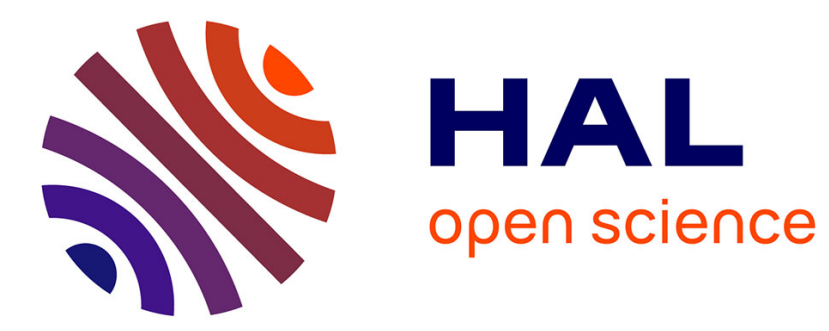

\title{
Key impact of phase dynamics and diamagnetic drive on Reynolds stress in magnetic fusion plasmas
} Y Sarazin, Guilhem Dif-Pradalier, X Garbet, P Ghendrih, Anatole Berger, C Gillot, V Grandgirard, K Obrejan, R Varennes, L Vermare, et al.

\section{- To cite this version:}

Y Sarazin, Guilhem Dif-Pradalier, X Garbet, P Ghendrih, Anatole Berger, et al.. Key impact of phase dynamics and diamagnetic drive on Reynolds stress in magnetic fusion plasmas. Plasma Physics and Controlled Fusion, 2021, 10.1088/1361-6587/abf673 . hal-03096598v2

\section{HAL Id: hal-03096598 \\ https://hal.science/hal-03096598v2}

Submitted on 2 Apr 2021

HAL is a multi-disciplinary open access archive for the deposit and dissemination of scientific research documents, whether they are published or not. The documents may come from teaching and research institutions in France or abroad, or from public or private research centers.
L'archive ouverte pluridisciplinaire HAL, est destinée au dépôt et à la diffusion de documents scientifiques de niveau recherche, publiés ou non, émanant des établissements d'enseignement et de recherche français ou étrangers, des laboratoires publics ou privés. 


\title{
Key impact of phase dynamics and diamagnetic drive on Reynolds stress in magnetic fusion plasmas
}

\author{
Y. Sarazin ${ }^{1}$, G. Dif-Pradalier ${ }^{1}$, X. Garbet ${ }^{1}$, P. Ghendrih ${ }^{1}$, \\ A. Berger ${ }^{1}$, C. Gillot ${ }^{1}$, V. Grandgirard ${ }^{1}$, K. Obrejan ${ }^{1}$, \\ R. Varennes $^{1}$, L. Vermare ${ }^{2}$ and T. Cartier-Michaud ${ }^{3}$ \\ 1 CEA, IRFM, Saint-Paul-lez-Durance, F-13108, France. \\ 2 LPP, CNRS, Ecole polytechnique, UPMC Univ Paris 06, Univ. Paris-Sud, \\ Université Paris-Saclay, Sorbonne Universités, 91128 Palaiseau, France. \\ 3 CERN, Route de Meyrin, Genève, Switzerland \\ E-mail: yanick.sarazin@cea.fr
}

2 April 2021

\begin{abstract}
Reynolds stress is a key facet of turbulence self-organization. In the magnetized plasmas of controlled fusion devices, the zonal flows that are driven by the averaged Reynolds stress modify the confinement performance. We address this problem with full- $f$ gyrokinetic simulations of ion temperature gradient driven turbulence. From the detailed analysis of the 3-dimensional electric potential and transverse pressure fields, we show that the diamagnetic contribution to the Reynolds stress - stemming from finite Larmor radius effects - exceeds the electrostatic contribution by a factor of about two. Besides, both contributions are in phase, indicating that pressure does not behave as a passive scalar. In addition, the Reynolds stress induced by the electric drift velocity is found to be mainly governed by the gradient of the phase of the electric potential modes rather than by their magnitude. By decoupling Reynolds stress drive and turbulence intensity, this property indicates a careful analysis of phase dynamics is crucial in the interpretation of experiments and simulations.
\end{abstract}

Keywords: turbulence, zonal flows, Reynolds stress, diamagnetic Reynolds stress, full- $f$ gyrokinetic simulations

Submitted to: Plasma Phys. Control. Fusion

\section{Introduction}

Since their discovery in numerical simulations as self-organization mediators of magnetized plasma turbulence [20,21], and since their identification as key players in the saturation of ion-scale turbulence in tokamak plasmas [22], the investigation 
of zonal flow generation is a major research effort aiming at its experimental and theoretical characterization (see the review [9] and references therein). In tokamaks, they correspond to one of the components of the flux surface averaged electric potential, hence resulting in a non-vanishing radial electric field profile. They usually appear at low frequency as compared to the underlying turbulence, in combination with the zero frequency mean radial electric field - stemming from the radial force balance in the plasma core [28] - and the high frequency geodesic acoustic modes [36] due to magnetic compressibility. By tearing apart turbulence eddies, such sheared flows are regulators of ion-scale turbulence and of the resulting transport [5]. As a matter of fact, the shear of the radial electric field is suspected to be the main trigger of the transition from low to high confinement regimes in tokamaks $[8,35]$, and to play an important role in internal transport barriers $[37,7]$.

From the experimental point of view, the precise role of zonal flows in these bifurcations remains a fruitful and open research area (cf. review [32]), in part due to their delicate experimental characterization (cf. review [15]). From the theoretical point of view, a critical question is their driving and damping mechanisms. Regarding the former, it was early acknowledged that the Reynolds stress is central in this process [10]. There, cross-correlations of the radial and poloidal components of the electric drift fluctuations, $\tilde{u}_{E r}$ and $\tilde{u}_{E \theta}$, may lead to a net momentum transfer from small to large scales, so that zonal flows - the predator - effectively feed on the underlying drift wave turbulence, the prey.

In the perspective of predicting the dynamics and magnitude of the Reynolds stress, and more generally how zonal flows nonlinearly saturate, two important issues are addressed in this paper. The first one deals with the weight of the diamagnetic component. Indeed, it appears that fluctuations of the diamagnetic flow $\tilde{u}_{r}^{\star}$ can also couple to $\tilde{u}_{E \theta}$ and drive a net poloidal momentum at large scale [33, 23]. This term is naturally present in the vorticity equation of fluid codes for plasma turbulence, since the diamagnetic velocity is one of the fluid drifts. In the gyrokinetic framework, it actually emerges from finite Larmor radius effects. To our knowledge, it has received little attention so far. It was reported to be sub-dominant with respect to its electric and more familiar counterpart shortly after the turbulence overshoot in a gyrokinetic simulation [13]. In the saturated regime of ion temperature gradient driven turbulence using the GYSELA code with adiabatic electrons [17], we find that it enhances the contribution of the electric drift velocity to the Reynolds stress by a factor of about two. The former characteristics reveals that pressure does not behave as a passive scalar in this case. The second issue addresses the role of the phase that governs the tilt of turbulence eddies. The phase - or tilting - instability is long known to be a possible mechanism to transform convection into sheared flow in ideal fluids [29]. More recently, the phase curvature was identified as the main component of the Reynolds force in a linear magnetized plasma device [38]. Here, we examine the possibility of driving the Reynolds stress solely from such a tilting instability, independently from the actual behavior of the turbulence intensity. Results from a reduced model of interchange 
turbulence are used to identify the mechanisms at play in the gyrokinetic simulation. In the latter, the Reynolds stress is found to be well correlated with the time dynamics of the phase - and not with turbulence intensity. Implications regarding the analysis of experimental data are discussed.

The paper is organized in the following way. Section 2 focuses on the diamagnetic part of the Reynolds stress, detailing its origin and characteristics in gyrokinetic simulations. The role of the phase gradient in the build up of the Reynolds stress is explored in section 3, both with a reduced model for interchange turbulence and in gyrokinetic simulations. Discussion and conclusion close the paper.

\section{Reynolds stress dominated by the diamagnetic contribution}

\subsection{The two components of the poloidal Reynolds stress}

The expression of the poloidal Reynolds stress emerges from the charge conservation equation, which governs the dynamics of the generalized vorticity. Its complete derivation and expression in the gyrokinetic framework, including all radial currents responsible for its time evolution, can be found in [26, 1], and more recently in [2]. In the present work, we only focus on the component governed by the advection by the $E \times B$ drift - then putting all other contributions in the $R H S$ term. Then, the dimensionless gyrokinetic equation reads as follows

$$
\partial_{t} \bar{f}+\{J[\phi], \bar{f}\}=R H S
$$

with the Poisson brackets defined by $\ddagger\{g, f\}=\frac{1}{r}\left[\partial_{r}\left(g \partial_{\theta} f\right)-\partial_{\theta}\left(g \partial_{r} f\right)\right]$. Here, $\bar{f}$ is the distribution function of the gyro-centers, $\phi$ the electric potential and $J[\phi]$ stands for the gyro-average operator applied to $\phi$ (strictly speaking, the gyro-average operator is $\langle J[\phi]\rangle_{v}$, with $\langle X\rangle_{v}=\int \mathrm{d}^{3} v X=\int_{0}^{+\infty} 2 \pi \mathrm{d} \mu \int_{-\infty}^{+\infty} \mathrm{d} v_{\|} X$ the integral over the velocity space). For the sake of simplicity, the inhomogeneity of the magnetic field is not retained in a first stage, so that grad- $B$ effects leading to turbulence equipartition are not accounted for. $B$ is then simply replaced by $B_{0}$, which is absorbed in normalizations. The latter are Larmor radius $\rho_{0}=m_{p} v_{T 0} / e B_{0}$ for length scales, cyclotron period $\omega_{c 0}^{-1}=m_{p} / e B_{0}$ for time scale and thermal velocity $v_{T 0}=\left(T_{0} / m_{p}\right)^{1 / 2}$ for velocities, where $m_{p}$ is the proton mass, $e$ is the elementary charge and $T_{0}$ a reference temperature. The electric potential is normalized by $T_{0} / e$. In the long wavelength limit, $J$ can be approximated by:

$$
J \approx 1+\frac{\mu}{2} \nabla_{\perp}^{2}
$$

where $\mu$ is the adiabatic invariant, namely the magnetic moment and $\nabla_{\perp}^{2}=\frac{1}{r} \partial_{r}\left(r \partial_{r}\right)+$ $\frac{1}{r^{2}} \partial_{\theta}^{2}$. Let us apply the gyro-average operator $J$ to eq.(1) and integrate over the velocity space: $\langle J[(1)]\rangle_{v}$. At this stage, one can use the quasi-neutrality constraint which can be

$\ddagger$ Notice that this definition of Poisson brackets uses an opposite sign convention to that often used in gyrokinetics. 
formulated as follows in the Boussinesq approximation:

$$
n_{0} \Omega=\langle J[\bar{f}]\rangle_{v} \approx\langle\bar{f}\rangle_{v}+\frac{1}{2} \nabla_{\perp}^{2} p_{\perp}
$$

where $\Omega=\phi-\langle\phi\rangle-\nabla_{\perp}^{2} \phi$ is the potential vorticity, with $\langle\phi\rangle \approx \iint \phi \mathrm{d} \theta \mathrm{d} \varphi / 4 \pi^{2}$ the flux surface averaged potential. The first term on the right hand side is the gyrocenter density, and the second one accounts for finite Larmor radius effects in the long wavelength limit, with $p_{\perp}=\langle\mu \bar{f}\rangle_{v}$ the transverse pressure. One then obtains, up to higher order terms in $\left(k_{\perp} \rho_{i}\right)^{2}$ :

$$
\partial_{t} \Omega-\left\{\phi, \nabla_{\perp}^{2} \phi+\frac{1}{2} \nabla_{\perp}^{2} p_{\perp}\right\}-\frac{1}{2}\left\{p_{\perp}, \nabla_{\perp}^{2} \phi\right\}-\frac{1}{2} \nabla_{\perp}^{2}\left\{p_{\perp}, \phi\right\}=\langle J[R H S]\rangle_{v}(4)
$$

Noticing that $\nabla_{\perp}^{2}\left\{\phi, p_{\perp}\right\}=\left\{\nabla_{\perp}^{2} \phi, p_{\perp}\right\}+\left\{\phi, \nabla_{\perp}^{2} p_{\perp}\right\}+2\left\{\nabla_{\perp, i} \phi, \nabla_{\perp, i} p_{\perp}\right\}$, with $\nabla_{\perp, i}$ the derivative along the $i^{\text {th }}$ Cartesian coordinate in the transverse plane and taking the sum on doubled indexes, eq.(4) can be recast as follows:

$$
\partial_{t} \Omega-\left\{\phi+p_{\perp}, \nabla_{\perp}^{2} \phi\right\}-\left\{\nabla_{\perp, i} p_{\perp}, \nabla_{\perp, i} \phi\right\}=\langle J[R H S]\rangle_{v}
$$

Equivalently, equation (5) also admits a conservative form:

$$
\partial_{t} \Omega-\nabla_{\perp, i}\left\{\phi+p_{\perp}, \nabla_{\perp, i} \phi\right\}=\langle J[R H S]\rangle_{v}
$$

Here, the Poisson bracket corresponds to the flux of vorticity. Further taking the flux surface average and integrating over the radial direction provides the evolution equation of the $E \times B$ poloidal flow $\left\langle u_{E \theta}\right\rangle=\partial_{r}\langle\phi\rangle$ :

$$
\frac{\partial\left\langle u_{E \theta}\right\rangle}{\partial t}+\frac{1}{r^{2}} \frac{\partial}{\partial r}\left[r^{2}\left(\pi+\pi^{\star}\right)\right]=r h s
$$

where the flux-surface averaged Reynolds stress (hereafter simply called Reynolds stress in short) and its diamagnetic component are given by:

$$
\begin{aligned}
\pi & =\left\langle\tilde{u}_{E r} \tilde{u}_{E \theta}\right\rangle \\
\pi^{\star} & =\left\langle\tilde{u}_{r}^{\star} \tilde{u}_{E \theta}\right\rangle
\end{aligned}
$$

The tilde refers to non axi-symmetric components, while $\tilde{u}_{E r}=-\frac{1}{r} \partial_{\theta} \tilde{\phi}$ and $\tilde{u}_{r}^{\star}=-\frac{1}{r} \partial_{\theta} \tilde{p}_{\perp}$ denote the radial components of the electric and diamagnetic drifts, respectively. As expected, the flux of vorticity is related to the Reynolds stress, which is a restatement of Taylor's theorem [16]. Also, it appears that finite Larmor radius effects, carried by the $J$ operator, lead to an additional contribution to the Reynolds stress, namely $\pi^{\star}$, as already acknowledged in early publications [33, 23]. Appendix A shows the consistency between this calculation within the gyrokinetic framework and the fluid moment approach in the adiabatic limit. Especially, the diamagnetic origin of $\pi^{\star}$ is assessed.

Hereafter, $\pi$ and $\pi^{\star}$ are named Reynolds stress and diamagnetic Reynolds stress, respectively. 


\subsection{Electric and diamagnetic contributions}

The structure and dynamics of the total Reynolds stress are explored in a flux-driven gyrokinetic simulation of the GYSELA code [17]. Ion temperature gradient (ITG) driven turbulence is modeled with an adiabatic electron response.

The simulation is similar to the one already reported in [11] (it is actually a rerun so as to get 3-dimensional data which are required in the present analysis). Its radial domain covers $0 \leq r / a \leq 1.3$, with a toroidal limiter treated as an immersed boundary where relaxation towards a low temperature Maxwellian is imposed by means of an additional Krook operator [6]. In the simulated scrape-off layer $r / a \geq 1$, the electron density relative fluctuation is clamped to the floating potential $\phi=3 T_{e} / e$. The $\rho^{\star}=\rho_{i} / a$ parameter is equal to $\rho^{\star} \approx 1 / 151$ and $\nu^{\star} \approx 7.510^{-2}$ at $r / a=0.5$.

We have also performed the same analysis on another well resolved simulation for which 3-dimensional output data were available. Its radial domain is $0 \leq r / a \leq 1$, the distribution function driven towards a Maxwellian at the outer edge, characterized by e-folding density and temperature profiles which mimic those expected in the scrapeoff layer of tokamak plasmas. Staircases are observed in the fully developed turbulent regime. In this case $\rho^{\star} \approx 1 / 244$ at $r / a=0.5$ where the collisionality is $\nu^{\star} \approx 0.23$. Results regarding the present issue are quite similar.
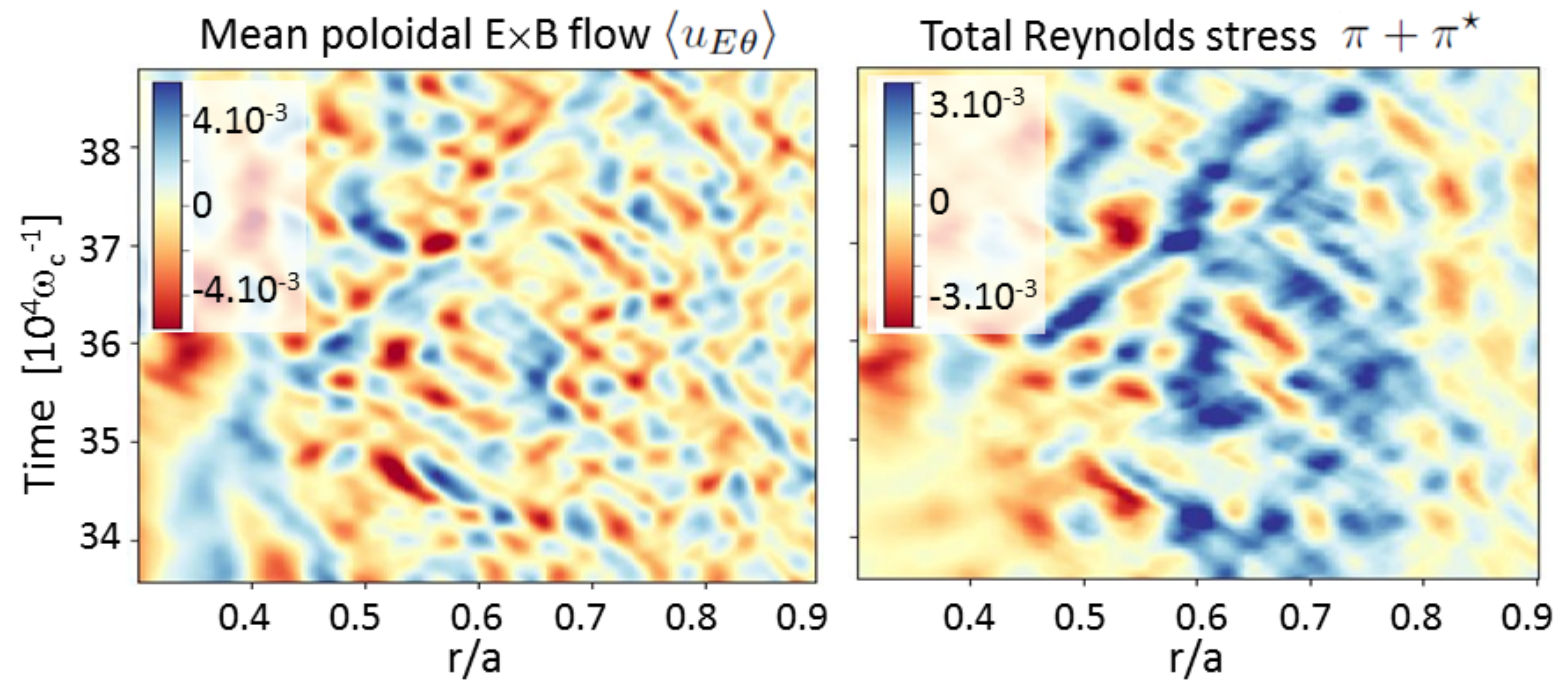

Figure 1. Time derivative of $\left\langle u_{E \theta}\right\rangle$ (left) and Reynolds force (right) versus normalized radius and time. The selected time interval is in the well saturated turbulent regime.

Figure 1 portraits the 2-dimensional behavior, in space and time, of the flux surface averaged $E \times B$ poloidal flow $\left\langle u_{E \theta}\right\rangle$ and of the total Reynolds stress $\pi+\pi^{\star}$. Two main observations can be made. First, the dynamics essentially features avalanche-like events which draw an array made of diagonal structures. These avalanches propagate both inwards and outwards, roughly at the same speed. Second, there appears to be 
some correlation between the two images, attesting the role of the total Reynolds stress in driving zonal flows. Deriving a vorticity equation which is tractable numerically is difficult in the gyrokinetic framework (see for instance the expressions proposed in $[26,1,2])$. We focus on the total Reynolds stress hereafter, bearing in mind its likely dominant contribution to the generation of dynamical corrugated features of the zonal radial electric field.

The respective contributions of the electric and diamagnetic flows to the Reynolds stress $\pi$ and $\pi^{\star}$ respectively are displayed on Fig.2. Two main observations can be made. First, $\pi$ and $\pi^{\star}$ are approximately in phase, minima and maxima occurring roughly at the same time and radial locations. Secondly, $\pi^{*}$ is about twice the value of $\pi$. This is shown on Fig.3, where each point corresponds to the values of $\pi$ and $\pi^{\star}$ at any given radial position $0.3 \leq r / a \leq 0.9$ (the radial domain excludes the central region where a non-vanishing heat source is applied and the outer buffer region) and time $335,700 \leq \omega_{c} t \leq 387,900$ (which corresponds to $347.88 \lesssim t v_{T i} / R_{0} \lesssim 402.02$, so well after the overshoot, into the nonlinear saturation phase). The important conclusion is that FLR corrections to the Reynolds stress tensor cannot be ignored, and even prove dominant in magnitude.

At this point, it is worth noticing that this result does neither imply nor mean that $\left(k_{\perp} \rho_{i}\right)^{2}$ terms are of order unity, which they are actually not as attested by the strong cut-off of the turbulence spectra above $k_{\perp} \rho_{i} \sim 1$ (see e.g. [25]). Phase shift plays there a critical role. Accounting for higher order terms in $\left(k_{\perp} \rho_{i}\right)^{2 m}(m \geq 2)$ would involve cross-correlations between fluctuations of the electric drift velocity and of high order derivatives of high order fluid moments.

The fact that $\pi^{\star}$ is found larger than $\pi$ is in marked difference with previous findings. To our knowledge, the only other observation in a gyrokinetic simulation was done by Dimits and coworkers [13]. They report a negligible role of the diamagnetic Reynolds stress in an ITG turbulence simulation for the cyclone base case. Apart from the possible issue with the definition of $\pi^{\star}$, there are several differences between this simulation and the ones reported in our paper which may result in these different findings. First of all, the one of Dimits is gradient-driven, so that the equilibrium pressure profile is kept constant over time conversely to our flux-driven simulations. It remains unknown how this constraint may impact the fluctuation dynamics and magnitude of the transverse pressure fluctuations and their gradient, which govern the diamagnetic Reynolds stress $\pi^{\star}$. Secondly, cyclone base-case gradient-driven simulations are close to the so-called Dimits upshift [12], i.e. close to the nonlinear threshold for turbulent transport. As reported e.g. in [27], they tend to be characterized by a secular growth of zonal flows, rendering early conclusions difficult to interpret. In that respect, and finally, Dimits'result is obtained in the early phase of the simulation: the time window which we have considered for the analysis starts at a time $t=33.5710^{4} \omega_{c}^{-1} \approx 2.10^{3} L_{T} / v_{T i}$ roughly equal to twice the final time of the simulation considered by Dimits. In the well saturated turbulent regime which we consider, Reynolds stress temporal fluctuations are 
much larger than their almost vanishing mean, conversely to what happens just after the turbulence overshoot in Fig.2(a) of [13]. In this same figure, the final state actually looks closer to our finding, especially in the sense that both Reynolds stress components tend to oscillate around a much smaller mean than their standard deviation, and that these standard deviations seem to reach comparable magnitudes, possibly in phase (although this latter point is more difficult to claim from the time traces). Incidentally, the analysis of the second simulation where we have 3-dimensional data from the linear phase reveals that the diamagnetic Reynolds stress is always twice as large as the Reynolds stress, even in the early turbulence regime from the overshoot onwards.

\subsection{Transverse pressure and potential fluctuations}

The approximate proportionality $\pi^{\star} \approx 2 \pi$ also indicates that the transverse pressure is not simply advected by the $\mathbf{E} \times \mathbf{B}$ flow. Indeed, assuming $\partial_{t} p_{\perp}+\mathbf{u}_{E} \cdot \boldsymbol{\nabla} p_{\perp}=0$, the linear analysis would lead to the following relation in the three-dimensional Fourier space: $\hat{p}_{\perp, m n \omega}=-\left(\omega_{p}^{\star} / \omega\right) \hat{\phi}_{m n \omega}$, with $\omega_{p}^{\star}=k_{\theta} \partial_{r}\left\langle p_{\perp}\right\rangle$ the diamagnetic frequency. Here, $m$ and $n$ are the poloidal and toroidal wave numbers, $k_{\theta}=m / r$ the poloidal wave vector and $\left\langle p_{\perp}\right\rangle$ the time and flux surface average of $p_{\perp}$. In this case, $\pi^{\star}=-\sum_{\omega, k} k_{r} k_{\theta}\left(\omega_{p}^{\star} / \omega\right)\left|\hat{\phi}_{m n \omega}\right|^{2}$ is expected to be out of phase with respect to $\pi=\sum_{\omega, k} k_{r} k_{\theta}\left|\hat{\phi}_{m n \omega}\right|^{2}$. This result could have important implications when interpreting experimental data (see e.g. [34]).

So as to look for the origin of the close to zero phase shift between $\pi$ and $\pi^{\star}$, the properties of the Fourier components of pressure and electric potential are investigated, see Fig.4. Only resonant modes are selected, i.e. such that $n+m / q(r) \approx 0$, since they are the main contribution to the Reynolds stress. The top panel shows that the amplitudes of the modes peak approximately at the diamagnetic frequency $\omega_{p}^{\star}$ (dot-dash black line). The bottom graph is a cut at $m=-45$. One recovers the ratio of about 2 between $\left|\hat{p}_{\perp m n \omega}\right|$ and $\left|\hat{\phi}_{m n \omega}\right|$. The relationship between $\hat{p}_{\perp m n \omega}$ and $\hat{\phi}_{m n \omega}$ is investigated on a systematic basis in Fig.5 which displays all data points of $\Re\left(\hat{p}_{\perp m n \omega}\right)$ as a function of $-\left(\omega_{p}^{\star} / \omega\right) \Re\left(\hat{\phi}_{m n \omega}\right), \Re$ denoting the real part. These are 3 -dimensional arrays, functions of $\omega, m$ and $r$. The range of $m$ values has been restricted to $-200 \leq m \leq 0$. The cloud of points does not exhibit any clear structure. Consequently, there is no particular trend indicating some form of correlation. If any, the sign between both quantities rather appears to be negative. In agreement with the result that $\pi$ and $\pi^{\star}$ are in phase, one can notice that $\Re\left(\hat{p}_{\perp m n \omega}\right)$ and $\Re\left(\hat{\phi}_{m n \omega}\right)$ have the same sign at large amplitude. Since the points are not aligned on the diagonal (black dashed line), this means that the fluctuations of $p_{\perp}$ are not simply advected by the $\mathbf{E} \times \mathbf{B}$ flow.

From the theoretical point of view, several mechanisms can explain the departure from the passive scalar hypothesis. Within the linear framework and from the fluid perspective, it was already noticed ([33], eq.(27) that compressibility could break the simple relationship $\hat{p}_{\perp, m n \omega}=-\left(\omega_{p}^{\star} / \omega\right) \hat{\phi}_{m n \omega}$. Also, considering the drift-kinetic (i.e. neglecting finite Larmor radius corrections) linear response, one obtains for the Fourier 

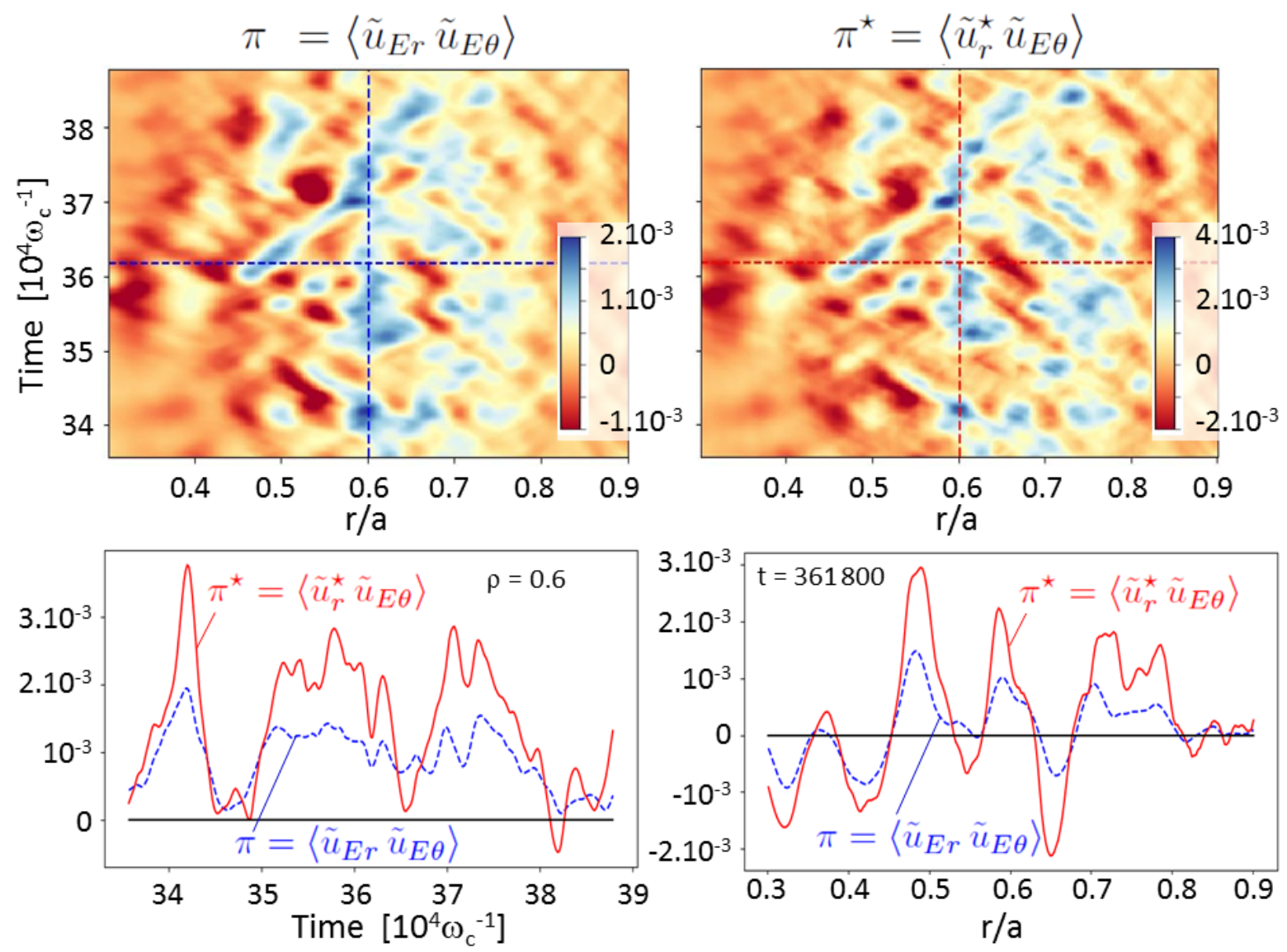

Figure 2. Two dimensional dynamics of $\pi(r, t)$ (left) and $\pi^{*}(r, t)$ (right). The colorscale is twice for $\pi^{*}$. Bottom: time evolution (left) at two different locations and radial profiles (right) at initial and final times.

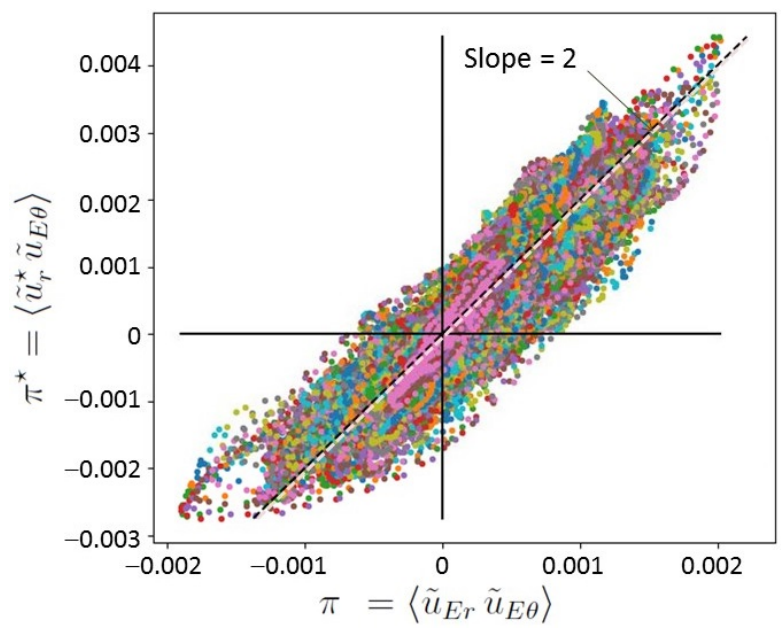

Figure 3. $\pi^{*}(r, t)$ as a function of $\pi(r, t)$. Each point corresponds to a value at a given time $t$ and location $r$. The slope of the dashed line is 2 . 
modes $\mathbf{k}, \omega$ (k standing for $m, n$ and $k_{r}$, the radial wave vector):

$$
\hat{p}_{\perp \mathbf{k}, \omega}=-\left\langle\frac{\omega^{\star}-\omega_{d}-k_{\|} v_{\|}}{\omega-\omega_{d}-k_{\|} v_{\|}} \mu B F_{M}\right\rangle_{v} \hat{\phi}_{\mathbf{k}, \omega}
$$

with $\omega_{d} \approx \mathbf{k} \cdot \mathbf{v}_{d}$ the frequency-operator associated to the vertical drift $\mathbf{v}_{d} \approx\left(m v_{\|}^{2}+\right.$ $\mu B)(\mathbf{b} \times \nabla B) / e B^{2}$ and $F_{M}$ the equilibrium Maxwellian distribution function. The brackets $\langle\ldots\rangle_{v}$ denote velocity-space integration. It readily appears that transverse pressure and electric potential deviate from the above-mentioned simple relationship due to both parallel dynamics $k_{\|} v_{\|}$and compressibility $\omega_{d}$. The simulation results reported here show that the usual assumption that their effect is negligible does not hold.

Looking for the possible leading terms in the above linear relationship, and/or whether it remains valid in the turbulent regime is certainly an important issue worth being addressed in future works.

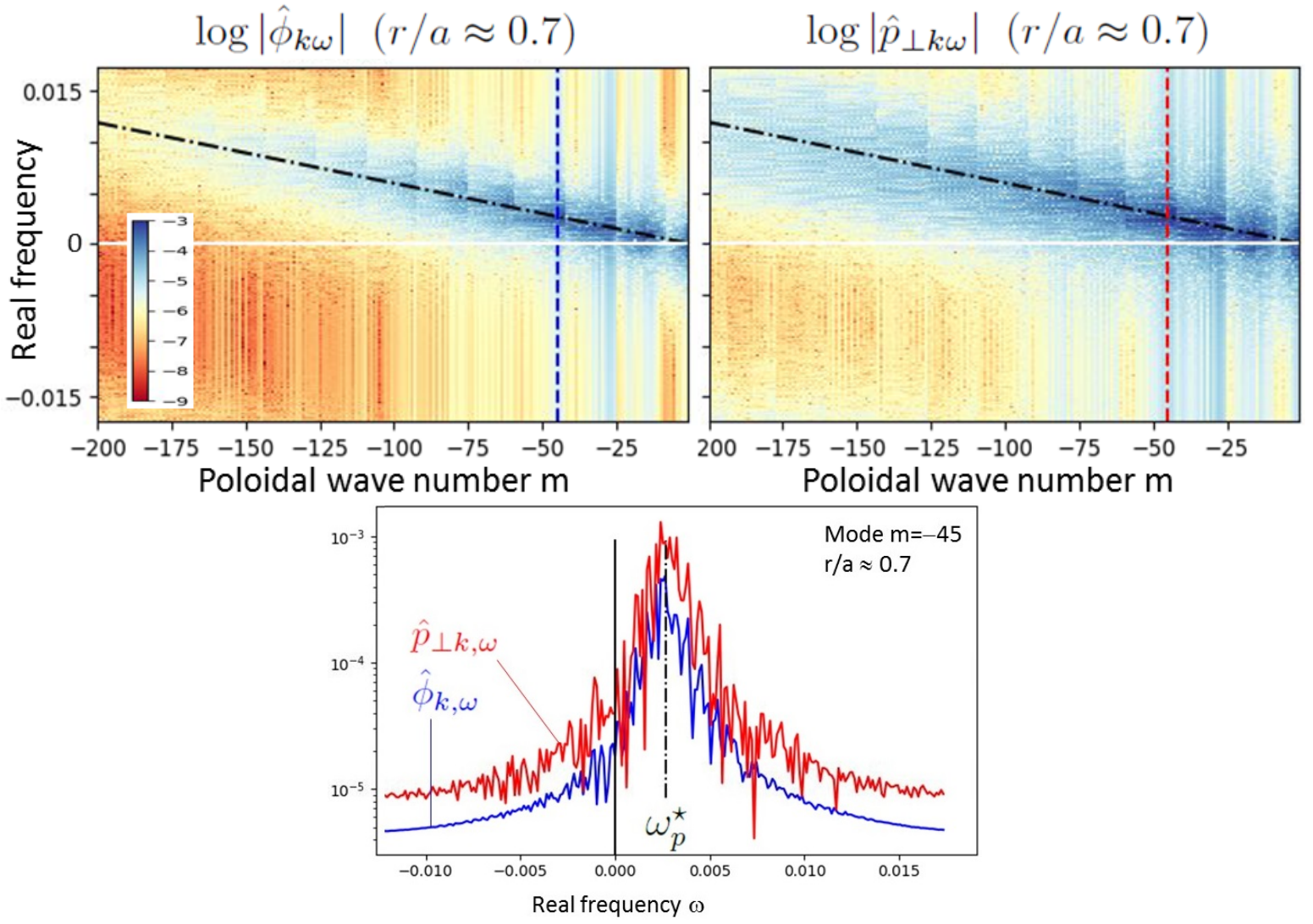

Figure 4. Top: amplitude (log scale) of $\hat{\phi}_{m n \omega}$ (left) and $\hat{p}_{\perp m n \omega}$ (right) as a function of poloidal mode number $m$ and frequency $\omega$ at $r \approx 175.7$. The toroidal mode number is such that $k_{\|} \approx 0$, i.e. $n \approx-m / q(r)$. The dotted-dashed line corresponds to the local diamagnetic frequency $\omega_{p}^{\star}$. Bottom: amplitude of $\hat{\phi}_{m n \omega}$ (blue) and $\hat{p}_{\perp m n \omega}$ (red) at $m=-45$. 


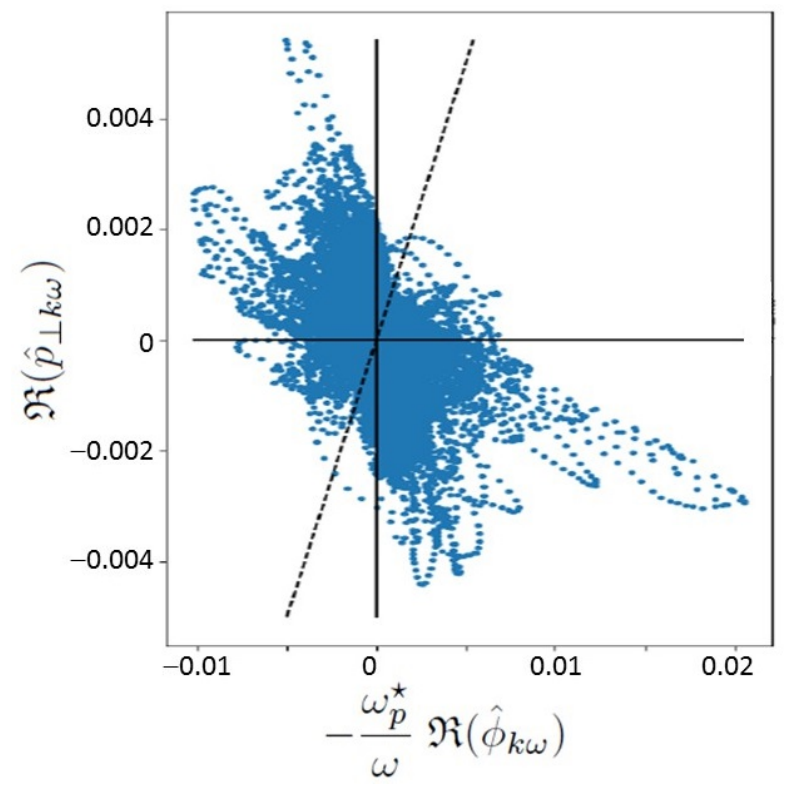

Figure 5. $\Re(Y)$ as a function of $\Re(X)$. $Y$ stands for $Y=\hat{p}_{\perp m n \omega}$ and $X$ for $X=-\left(\omega_{p}^{\star} / \omega\right) \hat{\phi}_{m n \omega}$.

\section{Reynolds stress: critical role of the phase}

\subsection{Role of phase and amplitude}

More insight can be gained into the structure of the Reynolds stress eqs.(8-9) by looking at their expression in the Fourier space. The electric potential $\phi$ and transverse pressure $p_{\perp}$ are decomposed in Fourier modes along the periodic directions, the poloidal $\theta$ and toroidal $\zeta$ angles (no Fourier transform in time is performed there):

$$
\begin{aligned}
& \phi=\sum_{m, n} \hat{\phi}_{m n} \mathrm{e}^{i(m \theta+n \zeta)}=\sum_{m, n}\left|\hat{\phi}_{m n}\right| \mathrm{e}^{i \varphi_{m n}^{\phi}} \mathrm{e}^{i(m \theta+n \zeta)} \\
& p_{\perp}=\sum_{m n} \hat{p}_{\perp, m n} \mathrm{e}^{i(m \theta+n \zeta)}=\sum_{m, n}\left|\hat{p}_{\perp, m n}\right| \mathrm{e}^{i \varphi_{m n}^{p}} \mathrm{e}^{i(m \theta+n \zeta)}
\end{aligned}
$$

with $\varphi_{m n}^{\phi}(r, t)$ and $\varphi_{m n}^{p}(r, t)$ the phase of the modes. Then $\pi$ and $\pi^{\star}$ can be recast as follows:

$$
\pi=-\frac{2}{r} \sum_{n} \sum_{m>0} m \Im\left(\hat{\phi}_{m n}^{*} \partial_{r} \hat{\phi}_{m n}\right)=-\frac{2}{r} \sum_{n} \sum_{m>0} m\left|\hat{\phi}_{m n}\right|^{2} \partial_{r} \varphi_{m n}^{\phi}
$$

and

$$
\begin{aligned}
\pi^{\star} & =-\frac{2}{r} \sum_{n} \sum_{m>0} m \Im\left(\hat{p}_{\perp, m n}^{*} \partial_{r} \hat{\phi}_{m n}\right) \\
& =-\frac{2}{r} \sum_{n} \sum_{m>0} m\left|\hat{\phi}_{m n}\right|\left|\hat{p}_{\perp, m n}\right| \\
& \times\left[\cos \Delta \varphi_{m n} \partial_{r} \varphi_{m n}^{\phi}+\sin \Delta \varphi_{m n} \partial_{r}\left(\ln \left|\hat{\phi}_{m n}\right|\right)\right]
\end{aligned}
$$


with $\Delta \varphi_{m n}=\varphi_{m n}^{\phi}-\varphi_{m n}^{p}$ the phase shift between the electric potential and transverse pressure fluctuations. $\Im$ denotes the imaginary part, and " $g$ " is the complex conjugate of $g$. Expression 11 highlights that both phase gradient and mode amplitude are key to have a non vanishing Reynolds stress $\pi$, as already stressed in early works [10]. Conversely, $\pi^{\star}$ can be generated by the gradient of either the phase or the amplitude depending on the phase shift $\Delta \varphi_{m n}$ between the two fields, which then governs the respective weight of each contribution.

\subsection{Phase instability in a reduced interchange model}

To highlight the role of the phase in the dynamics of the Reynolds stress, we first consider here the reduced nonlinear model proposed in [4] and recalled in Appendix B. It derives from the interchange instability, further considering flute modes $k_{\|}=0$ and a single poloidal wave vector denoted $k_{y}$. It extends the previous 1-dimensional model that features avalanche-like transport events [30] by keeping track of the phase of the density (or pressure since temperature is assumed constant) and electric potential fluctuations. Notice that the model was derived in the limit of cold ions, so that the diamagnetic Reynolds stress is not accounted for. On the basis of the results discussed in the previous section, the magnitude of the total Reynolds stress $\pi+\pi^{\star}-$ and possibly of the zonal flows - is expected to be enhanced when relaxing this assumption $T_{i} \approx 0$. For the time being, given the considered limit, the poloidal momentum equation reduces to $\partial_{t} V_{e q}=-\partial_{x} \pi$, with $V_{e q}=\left\langle u_{E \theta}\right\rangle$ the poloidal equilibrium flow and $x$ standing for the radial coordinate. The Reynolds stress $\pi$ takes the simple following form:

$$
\pi=-\Im\left(2 k_{y} \phi_{k}^{*} \partial_{x} \phi_{k}\right)
$$

with $\phi_{k}$ given equation (B.4). As expected from Taylor's identity, the Reynolds force is equal to the vorticity flux: $\partial_{x} \pi=-\Im\left(2 k_{y} \phi_{k}^{*} \partial_{x}^{2} \phi_{k}\right)$. An alternative form of $\pi$ can be obtained by introducing the magnitude and phase of the Fourier mode of the electric potential fluctuations: $\phi_{k}(x, t)=\left|\phi_{k}(x, t)\right| \exp \left[i \varphi_{\phi}(x, t)\right]$, with $\varphi_{\phi} \in \mathbb{R}$ the phase of the $\phi_{k}$ field. The Reynolds stress then reads

$$
\pi=-2 k_{y}\left|\phi_{k}\right|^{2} \partial_{x} \varphi_{\phi}
$$

The time evolution of the complex fluctuating fields $n_{k}$ (or $p_{k}$ since temperature is assumed constant) and $\phi_{k} \in \mathbb{C}$ can be re-expressed in terms of phase and amplitude dynamics as detailed in Appendix B.2.

Here, we compare two simulations which only differ by the magnitude $S_{0}$ of the source term. The values of the various parameters of eqs.(B.6) have been chosen close to the ones used in [4]: $N_{x}=256, L_{x}=1, \Delta t=2.10^{-3}, k_{y}=2 \pi, g=1$ and $D_{0}=D_{1}=\nu_{0}=\nu_{1}=6.510^{-2}$. The source term has a Gaussian shape:

$$
S=S_{0} \exp \left\{-\frac{(x-0.1)^{2}}{0.01}\right\}
$$


with $S_{0} \in\{20,30\}$. The boundary conditions are Dirichlet at $x=\{0, L\}$ for all fields, except for $n_{e q}$ at $x=0$ where we use Neumann: $\partial_{x} n_{e q}(x=0, t)=0$.

The time dynamics between the two cases $S_{0}=20$ and $S_{0}=30$ is different, as evidenced on fig. 6. For the small source magnitude, fluctuations first grow exponentially in time during the linear phase, and then saturate at a steady-state value. The system reaches a low confinement regime with large amplitude fluctuations and no equilibrium flow. Conversely, for $S_{0}=30$, the system bifurcates towards an improved confinement regime at $t \approx 75$. It appears that this transition is due to the generation of an equilibrium flow $V_{e q}$.

Most interestingly, it turns out that the build up of $V_{e q}$ results from an instability of the gradient of the phase of the electric potential $\partial_{x} \varphi_{\phi}$. Indeed, as shown on fig. 7, the phase gradient starts growing exponentially in time when the magnitude of the fluctuations reaches the first saturated regime. Actually, this governs the exponential growth of the Reynolds stress $\pi$ up to a second saturation regime, at lower amplitude $\left|\phi_{k}\right|$. Conversely, $\partial_{x} \varphi_{\phi}$ does not develop any instability and remains vanishing for $S_{0}=20$, resulting in a vanishing Reynolds stress .

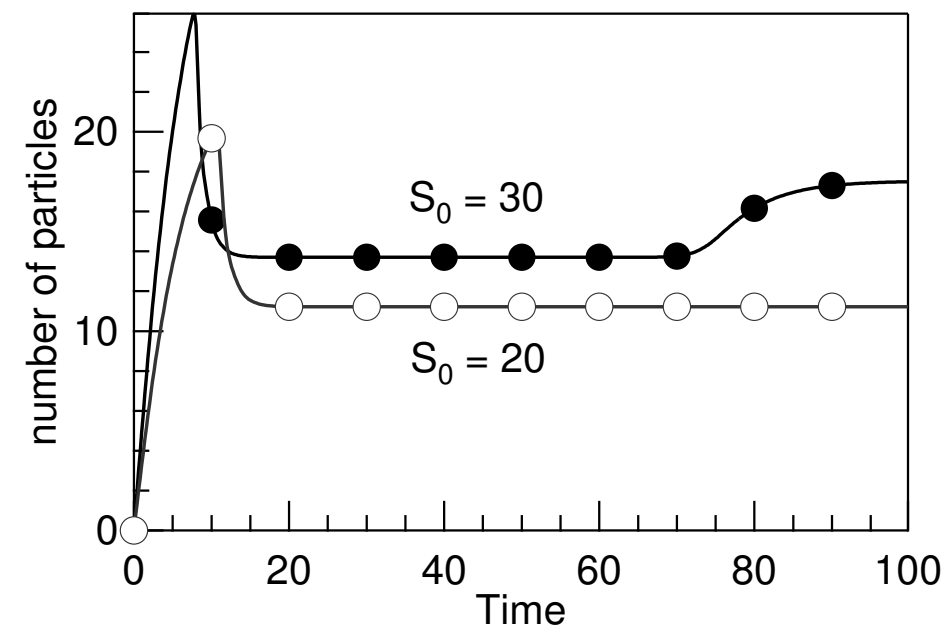

Figure 6. Time evolution of the total number of particles (also proportional to the internal energy since temperature is assumed constant) at 2 different values of the source magnitude.

Finding the expression of the growth rate of the phase instability is however not obvious. The expression of the time dynamics of the phase gradient $\partial_{x} \varphi_{\phi}$ is cumbersome, involving the equilibrium flow $V_{e q}$ and the cross-phase between the various fluctuating fields. Yet, one can gain some insight about the physics at work by first noticing that the gradient of the vorticity phase $\partial_{x} \varphi_{w}$ evolves according to $k_{y} \partial_{x} V_{e q}$ among other terms, eq.(B.18): $\partial_{t}\left(\partial_{x} \varphi_{w}\right)=-k_{y} \partial_{x} V_{e q}+\ldots$ This relation is reminiscent of the main expected effect of the velocity shear on turbulence eddies, namely to increase their radial wave vector $k_{x}: \mathrm{d} k_{x} / \mathrm{d} t=-k_{y} \partial_{x} V_{e q}$, with $k_{x} \approx \partial_{x} \varphi_{\phi}$ [9]. During the exponential growth, it 


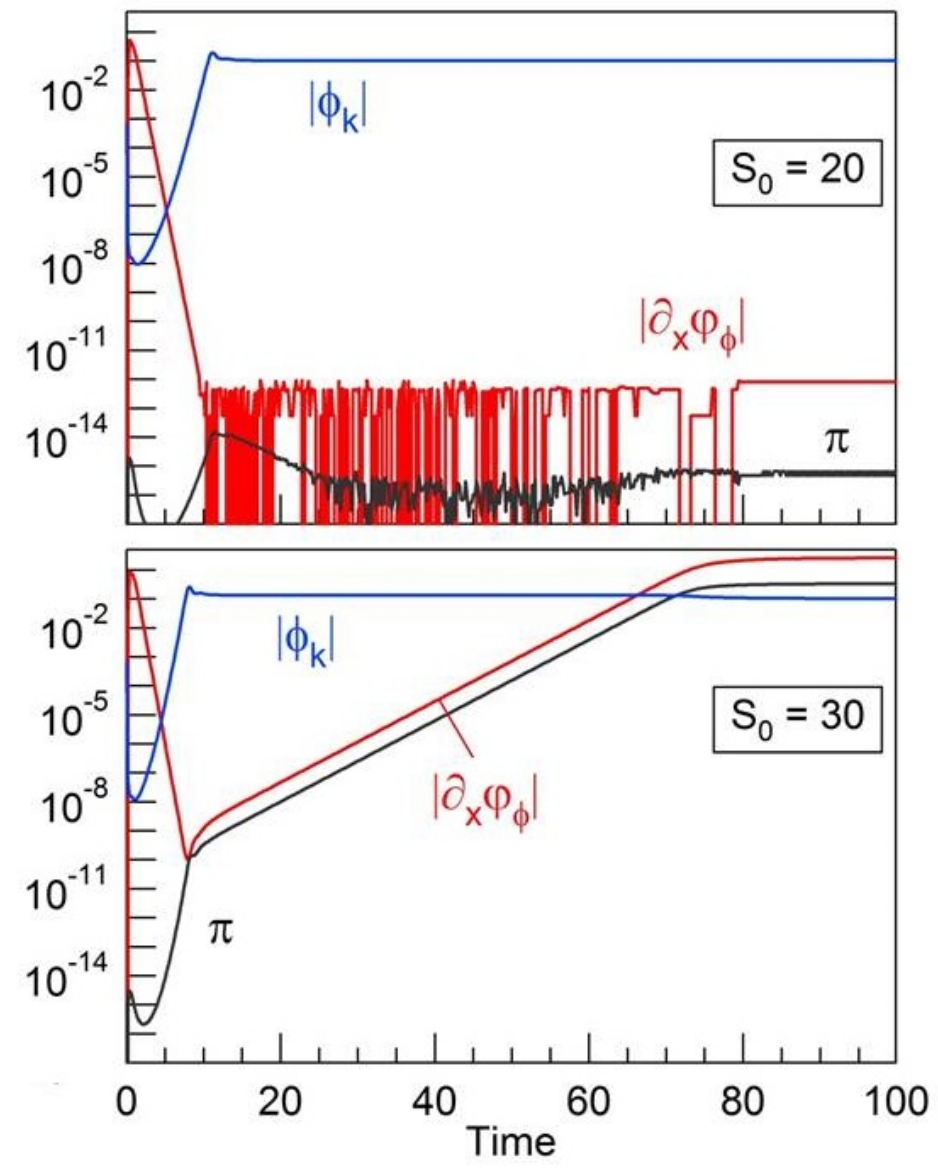

Figure 7. Time evolution of the Reynolds stress $\pi$ (black), of the gradient of the phase $\left|\partial_{x} \varphi_{\phi}\right|$ (red) and of the fluctuation magnitude $\left|\phi_{k}\right|$ at 2 different values of the source magnitude.

appears that all phase gradients exhibit the same growth rate, so that one can also infer $\partial_{t}\left(\partial_{x} \varphi_{\phi}\right)=-k_{y} \partial_{x} V_{e q}+\ldots$. Then, accounting for the momentum balance equation with the Reynolds stress given eq.(14) leads to:

$$
\partial_{t}^{2}\left(\partial_{x} \varphi_{\phi}\right) \approx-2 k_{y}^{2} \partial_{x}^{2}\left(\left|\phi_{k}\right|^{2}\right) \partial_{x} \varphi_{\phi}
$$

Dirichlet boundary conditions impose $\left|\phi_{k}\right|^{2}$ to be concave on average. Actually, with the chosen parameters, $\left|\phi_{k}\right|$ is broad scale and exhibits a single arch of sinusoid, so that $\partial_{x}^{2}\left(\left|\phi_{k}\right|^{2}\right)$ is negative everywhere. The positive expression $-2 k_{y}^{2} \partial_{x}^{2}\left(\left|\phi_{k}\right|^{2}\right)$ then provides a possible explanation for the observed growth rate, which would scale like $\gamma \approx \sqrt{2}\left|k_{y}\right|\left[\partial_{x}^{2}\left(\left|\phi_{k}\right|^{2}\right)\right]^{1 / 2} \sim \sqrt{2}\left|k_{y} k_{x} \phi_{k}\right|$. In this framework, the phase instability results from the self-reinforced shearing of turbulent eddies $\left(\partial_{t}\left(\partial_{x} \varphi_{\phi}\right)\right.$ term) by the Reynolds stress. In the course of the above derivation of the growth rate, we have assumed that the curvature of the Reynolds stress is the dominant term, i.e. $\partial_{x}^{2}\left(\left|\phi_{k}\right|^{2} \partial_{x} \varphi_{\phi}\right) \approx$ $\partial_{x}^{2}\left(\left|\phi_{k}\right|^{2}\right) \partial_{x} \varphi_{\phi}$. Although the detailed analysis of the simulation results reveals that this approximation is marginally valid, it turns out that $\partial_{x}^{2}\left(\left|\phi_{k}\right|^{2} \partial_{x} \varphi_{\phi}\right)$ and $\partial_{x}^{2}\left(\left|\phi_{k}\right|^{2}\right) \partial_{x} \varphi_{\phi}$ exhibit a similar shape, hence providing confidence in the qualitative expression of $\gamma$. 
This instability is also known as the tilting instability which transforms convection into sheared flow in 2-dimensional ideal fluids [29]. It is responsible for the generation of zonal flows by large amplitude drift (or Rossby) waves governed by the CharneyHasegawa-Mima equation. In this case, it was shown to exist above a finite amplitude threshold of the electric potential [19]. Consistently, the different dynamical regimes characterizing the 1D model are in agreement with the existence of a threshold, the instability only developing above a critical magnitude $S_{0, \text { crit }}$ of the driving source term $S_{0}$, in between $20<S_{0, \text { crit }}<30$ (cf. fig.7). More recently, the possible role of the tilting instability in the bifurcation towards H-mode in tokamak plasmas has been addressed [14].

In the next section, the evidence of such a phase instability is reported in the saturated regime of flux-driven ITG turbulence.

\subsection{The phase gradient and Reynolds stress growth in gyrokinetic simulations}

So as to identify the respective role of phase gradient and mode amplitude in the time dynamics of the Reynolds stress $\pi$, equation (11) is recast as follows:

$$
\begin{aligned}
\pi(r, t)=\mathcal{I}(r, t) & \varphi^{\prime}(r, t) \Delta(r, t) \\
\text { with } \quad \mathcal{I}(r, t) & =\sum_{n} \sum_{m>0} \frac{2 m}{r}\left|\hat{\phi}_{m n}\right|^{2} \\
\varphi^{\prime}(r, t) & =-\sum_{n} \sum_{m>0} W_{m n} \partial_{r} \varphi_{m n}^{\phi}
\end{aligned}
$$

Here, $\mathcal{I}$ captures the turbulence intensity. $W_{m n}(r)=\left\langle\left|\pi_{m n}\right|\right\rangle_{t} /\langle\pi\rangle_{t}$ - with $\pi_{m n}$ defined by the implicit expression $\pi(r, t)=\sum_{n} \sum_{m>0} \pi_{m n}(r, t)$ - is the normalized time-averaged radial profile of the $(m, n)$ spectrum of the Reynolds stress $\pi$. This weight aims at discarding contributions $\partial_{r} \varphi_{m n}^{\phi}$ to the phase gradient $\varphi^{\prime}(r, t)$ of $(m, n)$ modes that are far from the resonance condition $k_{\|}=0$, i.e. $n+m / q \approx 0$. The weight $W_{m n}(r)$ at $r \approx 180$ is displayed on Fig.8. The width of $W_{m n}$ partly accounts for the fact that GYSELA uses the geometrical angle $\theta$ as poloidal coordinate, so that the resonant condition slightly departs from $n+m / q=0$. Finally, $\Delta(r, t)$ accounts for the mismatch between $\pi$ and the product $\mathcal{I} \varphi^{\prime}$. Note that $|\Delta|$ can be larger than unity due to the use of the weight function in the definition of $\varphi^{\prime}$.

The question is whether $\pi$ is primarily governed by turbulence intensity $\mathcal{I}$ or by the phase gradient $\varphi^{\prime}$. To this end, we perform the cross-correlation in time between the Reynolds stress $\pi$ and $\varphi^{\prime}$ on the one hand, and $\pi$ and $\mathcal{I}$ on the other hand. Both are plotted at each radial location in Fig.9. They are computed during the same time window of saturated turbulence reported in the previous section 2. It appears that $\varphi^{\prime}$ exhibits a large correlation with $\pi$, up to 0.77 at vanishing time lag. Conversely, at most of the radial positions, the cross-correlation between $\pi$ and $\mathcal{I}$ remains small or even negative§. An example of the time dynamics of these three - rescaled - quantities

$\S$ Noticeably, it appears that the cross-correlation $\pi$-I is large when that of $\pi-\varphi^{\prime}$ is lost, e.g. at $0.3<r / a<0.35$. 


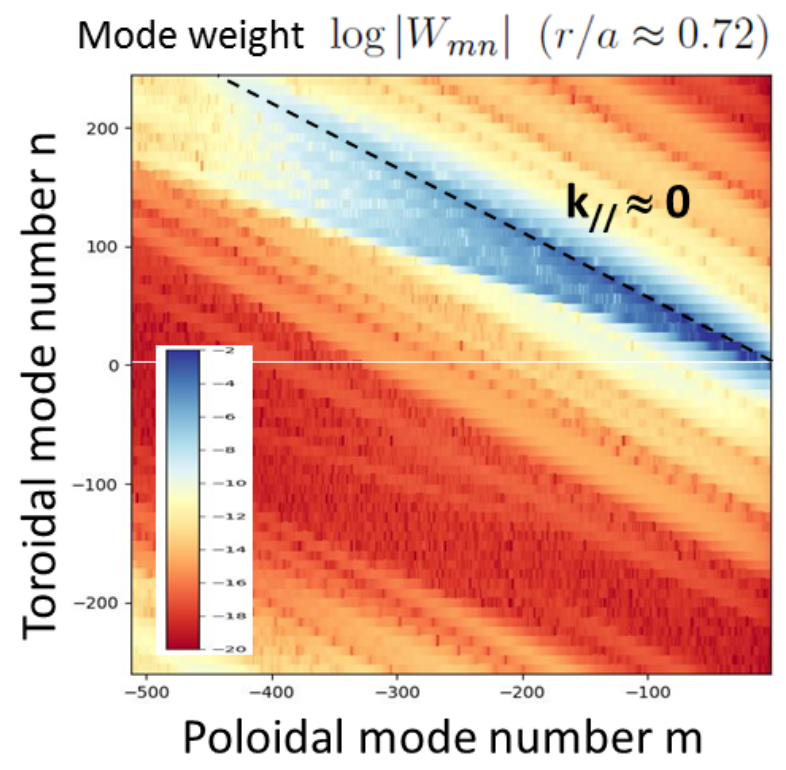

Figure 8. Weight function $W_{m n}$ - in logarithmic scale - computed at $r \approx 180$, as a function of the toroidal $n$ (x-axis) and poloidal $m$ (y-axis) wave numbers.

is plotted on Fig.10, at $r \approx 180(r / a \approx 0.72)$. We get the confirmation that both the increase and decay of $\pi$ are well correlated with those of the phase gradient $\varphi^{\prime}$. Even sometimes, e.g. at $t \sim 3510^{4}$ and $t \sim 36.710^{4}, \pi$ is growing while the amplitude contribution is decreasing. In this case, $\pi$ is only driven by the growth of the phase gradient.

The critical role of the gradient of the phase in the triggering of finite poloidal flow has already been acknowledged in the literature [18]. There, from a heuristic model, the authors emphasize that a finite phase curvature alone - i.e. even if turbulence is homogeneous (which translated into $\partial_{r}\left|\hat{\phi}_{m n}\right|^{2}=0$ in our notations) - can generate a finite Reynolds force. Such an effect has been later reported in experimental measurements in a linear plasma device [38]. The properties of the Reynolds stress in the gyrokinetic simulations which we report here extend these predictions and observations in the temporal space, in the sense that the time evolution of $\pi$ can be entirely governed by that of $\varphi^{\prime}$, even if the turbulence intensity $\mathcal{I}$ remains constant in time.

This result has important implications regarding experimental measurements. Indeed, one of its consequences is that the time dynamics of the Reynolds stress cannot be inferred from that of the amplitude of density - or electric potential - fluctuations only. Indeed, our analysis has shown that $\pi$ and $\mathcal{I}$ are weakly correlated in general, and can event exhibit anti-correlation. In particular, even though the magnitude of the electric potential fluctuations would be roughly constant, this would not necessarily imply that the magnitude of the Reynolds stress - hence possibly of the zonal flows - remains constant either. Conversely, both can actually exhibit opposite trends, as observed sometimes in figure 10. Our results provide the first evidence from gyrokinetic simulations that the time variation of the Reynolds stress is mainly governed by the 
phase gradient dynamics, rather than by the magnitude of turbulent fluctuations.

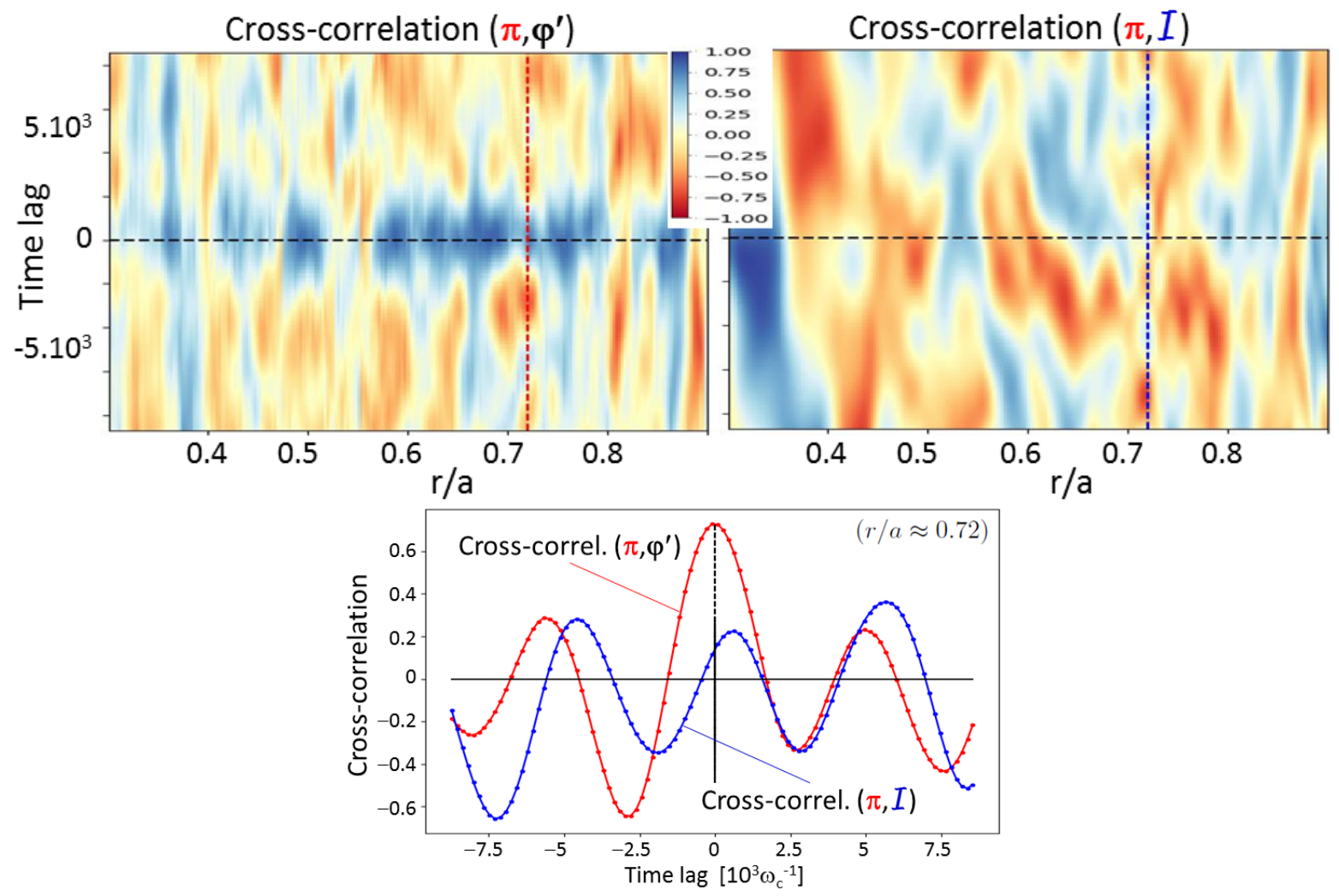

Figure 9. Top panel: cross-correlation in time, at each radial position, between $\delta \pi$ with $\delta \varphi^{\prime}$ (left) and $\delta \mathcal{I}$ (right), with $\delta X=X-\langle X\rangle_{t}$. Lower panel: same data at $r / a \approx 0.72$.

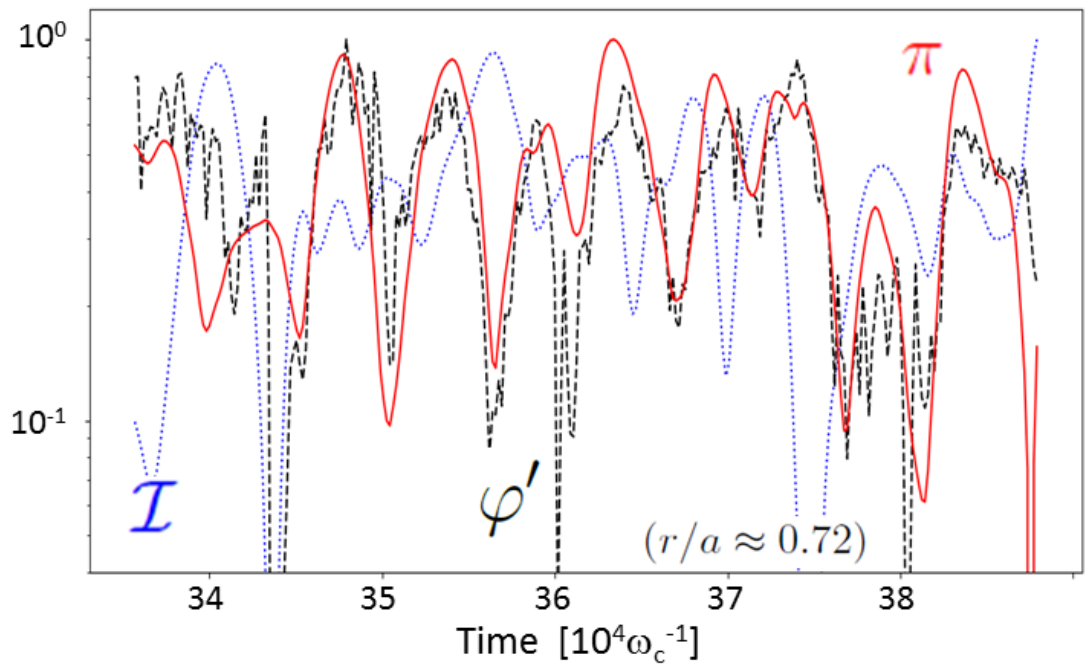

Figure 10. Time evolution of $\tilde{\pi}$ (red), $\tilde{\varphi}^{\prime}$ (green) and $\tilde{\mathcal{I}}$ (blue), with $\tilde{X}=1-$ $X / \max _{t}(X)$ the fluctuation relative to the maximum at $r / a \approx 0.72$. 


\section{Discussion and conclusion}

The poloidal Reynolds stress is the centerpiece of zonal flow generation. Given the key role of the latter in the regulation of ion-scale turbulence in tokamak plasmas, understanding its structure and dynamics appears essential at least for two reasons. On the one hand, this opens the door to upgraded nonlinear reduced transport models and experimental analyses. On the other hand, this may help tokamak plasma operation

by possibly offering the controlled access to improved confinement regimes. In this framework, two main results have been reported in this paper, on the basis of the analysis of 3-dimensional fluctuation data in gyrokinetic simulations of saturated ITG turbulence (with adiabatic electrons) with the GYSELA code. First, the diamagnetic component of the Reynolds stress is found to be in phase with the one governed by the electric drift, and to be roughly twice as large. This means that the transverse pressure cannot be viewed as a passive scalar in this regime, and that the two components have to be computed to properly quantify the source magnitude of zonal flows. Second, while the Reynolds stress can be roughly split in the product of turbulence intensity times a phase term, it appears that the latter largely dominates its dynamics. Indeed, the phase gradient is strongly cross-correlated with the Reynolds stress, while such a feature is less pronounced with the turbulence intensity. The mechanism is reminiscent of the tilting instability, which is highlighted in a reduced model for interchange turbulence. By decoupling the temporal dynamics of the turbulence intensity from that of the Reynolds stress, this property has profound implications on the interpretation of turbulence experimental measurements.

From the simulation side, the pending questions are twofold. First, what is the role of the diamagnetic Reynolds stress and the phase instability in triggering transport barriers, either a single strong barrier as the H-mode or multiple and comparatively weak as with staircases? Second, how do these features evolve in the presence of kinetic electrons, and for trapped electron mode (TEM) turbulence?

From the experimental point of view, assessing the impact (i) of the phase dynamics and (ii) of the diamagnetic drive on the total Reynolds stress is likely more challenging. The former (i) should be accessible via Langmuir probe array measurements at the edge of tokamak plasmas or in other laboratory devices. The latter (ii) is more delicate owing to the necessity of measuring both electric potential and pressure fluctuations at the same location. Its importance might be indirectly inferred from the possible imbalance between the divergence of the Reynolds stress $\left\langle\tilde{u}_{E r} \tilde{u}_{E \theta}\right\rangle$ and the time derivative of the zonal flows $\left\langle u_{E \theta}\right\rangle$ - although other contributions are also expected. Also, regimes with lower magnitude pressure fluctuations are expected to have a lower Reynolds stress drive, hence reduced zonal flow activity. As a final remark, one can notice that retrieving these characteristics in the hot core of tokamak plasmas - hence in the parameter regime where they have been reported in this paper - may likely reveal inaccessible with current diagnostics. Indirect signatures may then be looked for for their validation. In that respect, if the Reynolds stress components are found in numerical simulations to exhibit distinct features in ITG and TEM dominated turbulence, such changes could 
be detectable experimentally. In any case, our results add to the growing evidence that zonal flow generation is possible even at fixed turbulence intensity.

\section{Acknowledgments}

The authors warmly thank (in alphabetical order) Y. Asahi, P.H. Diamond, N. Fedorczak, Z. Guo, Ö. Gürcan, P. Hennequin and D. Zarzoso for stimulating discussions, and Ch. Passeron for her invaluable support. This work has also greatly benefited from discussions at the tenth Festival de Théorie in Aix-en-Provence (2019, France) and at the first Chengdu Theory Festival (2018, China). This research was supported in part by the National Science Foundation under Grant No. NSF PHY-1748958 during the KITP program on "Layering in Atmospheres, Oceans and Plasmas" (2021). Last, we are pleased to acknowledge the comments of an anonymous referee, which have lead to the developments found in Appendix A. The work has been carried out within the framework of the EUROfusion Consortium and was supported by the EUROfusion Theory and Advanced Simulation Coordination (E-TASC) initiative under the TSVV (Theory, Simulation, Verification and Validation) "L-H transition and pedestal physics" project (2019-2020). It has also received funding from the Euratom research and training program. The project has received funding from the European Unions Horizon 2020 research and innovation program under grant agreement No 824158 (EoCoE-II). The views and opinions expressed herein do not necessarily reflect those of the European

Commission. This work was also granted access to the HPC resources of TGCC and CINES made by GENCI, and to the EUROfusion High Performance Computer Marconi-Fusion. We acknowledge PRACE for awarding us access to Joliot-Curie at GENCI@CEA, France and MareNostrum at Barcelona Supercomputing Center (BSC), Spain.

\section{Appendix A. $\pi^{\star}$ from the fluid moment perspective}

The aim of this appendix is to make explicit the link between $\pi^{\star}$ derived within the gyrokinetic framework (eq.(9)) and the fluid moment approach. Especially, the diamagnetic origin of $\pi^{\star}$ is assessed.

In the adiabatic limit where the magnetic field evolves slowly as compared to the cyclotron period and on large scales as compared to the cyclotron radius, the transverse projection of the momentum balance equation stemming from the fluid moment approach can be treated order by order in the small expansion parameter $\varepsilon \sim \omega / \omega_{c i} \sim \rho_{i} / R \ll 1$, with $\omega$ and $\omega_{c i}$ the characteristic frequency of the problem and the ion cyclotron frequency, and $\rho_{i}$ and $R$ the thermal ion Larmor radius and major radius.

At leading order $\mathcal{O}(\varepsilon)$, the two drift velocities are the electric drift $\mathbf{u}_{E}$ and the diamagnetic drift $\mathbf{u}_{i}^{*}$ :

$$
\mathbf{u}_{\perp}^{(1)}=\mathbf{u}_{E}+\mathbf{u}_{i}^{*}=\frac{\mathbf{B} \times \nabla \phi}{B^{2}}+\frac{\mathbf{B} \times \nabla p_{\perp}}{e n B^{2}}
$$


Here, small correction terms proportional to the pressure anisotropy $\left(p_{\|}-p_{\perp}\right)$ have been neglected in the expression of the diamagnetic drift. This simplification is even more valid in the present derivation since these terms originate from the inhomogeneity of the magnetic field, which is neglected here. At next order $\mathcal{O}\left(\varepsilon^{2}\right)$, the polarization drift emerges. Accounting for the gyroviscous cancellation [24], it takes the following form:

$$
\mathbf{u}_{\perp}^{(2)}=\mathbf{u}_{p o l} \approx-\frac{m_{i}}{e B^{2}}\left[\partial_{t}+\left(\mathbf{u}_{E}+\mathbf{u}_{i}^{*}\right) \cdot \nabla\right] \boldsymbol{\nabla}_{\perp} \phi
$$

Due to the mass scaling, the electron polarization drift can be safely ignored. Now, consider the charge conservation equation $\boldsymbol{\nabla} . \mathbf{j}=0$. When neglecting the inhomogeneity of the magnetic field as we did in section 2, the divergence of the diamagnetic current vanishes. Therefore, the divergence of transverse currents reduces to $\boldsymbol{\nabla} \cdot \mathbf{j}_{\text {pol }}$, leading to (still using the Boussinesq approximation):

$$
\begin{aligned}
\boldsymbol{\nabla} \cdot \mathbf{j}_{p o l} \approx & -\frac{n m_{i}}{B^{2}}\left(\partial_{t}+\mathbf{u}_{E} \cdot \nabla\right) \nabla_{\perp}^{2} \phi-\frac{m_{i}}{e B^{3}} \nabla \cdot\left[\left(\frac{\mathbf{B} \times \nabla_{\perp}}{B} \cdot \nabla\right) \nabla_{\perp} \phi\right] \\
\approx & -\frac{n m_{i}}{B^{2}}\left(\partial_{t} \nabla_{\perp}^{2} \phi+\frac{1}{B}\left\{\phi, \nabla_{\perp}^{2} \phi\right\}\right) \\
& -\frac{m_{i}}{e B^{3}}\left(\left\{\nabla_{\perp, i} p_{\perp}, \nabla_{\perp, i} \phi\right\}+\left\{p_{\perp}, \nabla_{\perp}^{2} \phi\right\}\right)
\end{aligned}
$$

Up to normalization factors, the divergence of the polarization current eq.(A.3) turns out to be equivalent to the left hand side of eq.(5) within the same approximation of constant magnetic field. Therefore, it readily appears that the pressure terms in the general expression of Reynolds tensor, namely $\pi^{\star}$, originate from the ion diamagnetic drift which appears in the expression of the polarization drift, eq.(A.2). Notice that the charge dependence of the diamagnetic drift - leading to opposite signs for electron and ions - can be safely ignored here since the polarization drift of ions only is worth being considered.

Last, it should not be a surprise that finite Larmor radius effects within the gyrokinetic framework be related to contributions of the diamagnetic velocity. Indeed, this fluid drift itself actually results from such finite Larmor radius effects, namely the finite excursion of particle trajectories in the transverse plane due to the cyclotron motion.

\section{Appendix B. Reduced interchange model}

\section{Appendix B.1. Derivation of the model}

We start from the following minimal system for electrostatic interchange turbulence, reduced to 2 -dimensions by considering flute modes $k_{\|}=0$. It involves density $n$ and electric potential $\phi$, coupled together via the continuity and charge conservation equations:

$$
\begin{aligned}
& \partial_{t} n+[\phi, n]=S_{n}+\nabla_{\perp} \cdot\left(D \nabla_{\perp} n\right) \\
& \partial_{t} w+[\phi, w]+g \partial_{y} \log n=\nabla_{\perp \cdot} \cdot\left(\nu \nabla_{\perp} w\right) \\
& w=\nabla_{\perp}^{2} \phi
\end{aligned}
$$


Distances are normalized to the ion thermal Larmor radius $\rho_{c}=\left(m_{i} T\right)^{1 / 2} / e B((x, y) \rightarrow$ $\left.(x, y) / \rho_{c}\right)$, and time to the ion cyclotron frequency $\omega_{c}=e B / m_{i}\left(t \rightarrow \omega_{c} t\right)$, with $e$ the elementary charge, $T$ and $B$ being the constant electron temperature (cold ions are considered) and magnetic field. Finally, $g \sim \rho_{c} / R$ accounts for the average curvature of the magnetic field lines [31]. Each field is split in equilibrium and fluctuating parts. A single poloidal ( $y$ direction) mode $k$ is then retained for the fluctuating fields:

$$
\begin{aligned}
& n(x, y, t)=n_{e q}(x, t)+n_{k}(x, t) \mathrm{e}^{i k y}+n_{k}^{*}(x, t) \mathrm{e}^{-i k y} \\
& \phi(x, y, t)=\phi_{e q}(x, t)+\phi_{k}(x, t) \mathrm{e}^{i k y}+\phi_{k}^{*}(x, t) \mathrm{e}^{-i k y} \\
& w(x, y, t)=w_{e q}(x, t)+w_{k}(x, t) \mathrm{e}^{i k y}+w_{k}^{*}(x, t) \mathrm{e}^{-i k y}
\end{aligned}
$$

Equilibrium ( $y$-averaged) quantities $n_{e q}, \phi_{e q}$ and $w_{e q}$ are real $(\in \mathbb{R})$, while $n_{k}, \phi_{k}$ and $w_{k}$ are complex fields $(\in \mathbb{C})$. This approach is in marked contrast with the derivation

of reference [30], where the phase shift between density and potential fluctuations was assumed to be clamped to $\pi / 2$, hence maximizing turbulent transport. Introducing the equilibrium velocity:

$$
V_{e q}=\partial_{x} \phi_{e q}
$$

one finally obtains the following 4-field system of equations:

$$
\begin{aligned}
& \partial_{t} n_{e q}=2 k \partial_{x}\left[\Im\left(n_{k} \phi_{k}^{*}\right)\right]+\partial_{x}\left(D_{0} \partial_{x} n_{e q}\right)+S \\
& \partial_{t} V_{e q}=2 k \partial_{x}\left[\Im\left(\phi_{k}^{*} \partial_{x} \phi_{k}\right)\right]+\partial_{x}\left(\nu_{0} \partial_{x} V_{e q}\right) \\
& \partial_{t} n_{k}=i k\left(\phi_{k} \partial_{x} n_{e q}-V_{e q} n_{k}\right)-D_{1} k^{2} n_{k}+\partial_{x}\left(D_{1} \partial_{x} n_{k}\right) \\
& \partial_{t} w_{k}=i k\left(\phi_{k} \partial_{x}^{2} V_{e q}-V_{e q} w_{k}-g \frac{n_{k}}{n_{e q}}\right)-\nu_{1} k^{2} w_{k}+\partial_{x}\left(\nu_{1} \partial_{x} w_{k}\right) \\
& w_{k}=\left(\partial_{x}^{2}-k^{2}\right) \phi_{k}
\end{aligned}
$$

The turbulent particle flux and the Reynolds stress then read:

$$
\begin{aligned}
& \Gamma=-2 k \Im\left(n_{k} \phi_{k}^{*}\right) \\
& \pi=-2 k \Im\left(\phi_{k}^{*} \partial_{x} \phi_{k}\right)
\end{aligned}
$$

Alternative forms of the Reynolds force may reveal more appropriate for numerical schemes: $\partial_{x} \pi=-2 k \Im\left(\phi_{k}^{*} w_{k}\right)=-2 k \Im\left(\phi_{k}^{*} \partial_{x}^{2} \phi_{k}\right)$.

The system B.6 was initially derived in [4]. Notice that the system proposed in [3] differs from this one by neglecting the radial curvature of the potential with respect to its poloidal one, i.e. assumes $\left|k^{2} \phi_{k}\right| \gg\left|\partial_{x}^{2} \phi_{k}\right|$. In this case, vorticity and potential are in phase. Actually, this latter system reveals numerically unstable, leading to the transient excitation of fluctuations at spatial scales down to the grid size.

\section{Appendix B.2. Amplitude and phase equations}

An alternative form of the previous system can be derived by introducing the magnitude and phase of the retained Fourier component $k$ of the fluctuations.

$$
\hat{f}(x, t)=A_{f}(x, t) e^{i \varphi_{f}(x, t)}
$$


with $A_{f} \equiv|\hat{f}| \geq 0$ and $\varphi_{f} \in \mathbb{R}$. One further introduces the following phase shifts (for any $f, g \in n, \phi, w)$ :

$$
\Delta \varphi_{f g} \equiv \varphi_{f}-\varphi_{g}
$$

System (B.6) can then be recast in terms of amplitudes and phases. The equations for the mean quantities read:

$$
\begin{aligned}
& \partial_{t} n_{e q}=2 k \partial_{x}\left(A_{n} A_{\phi} \sin \Delta \varphi_{n \phi}\right)+\partial_{x}\left(D_{0} \partial_{x} n_{e q}\right)+S_{n} \\
& \partial_{t} V_{e q}=2 k \partial_{x}\left(A_{\phi}^{2} \partial_{x} \varphi_{\phi}\right)+\partial_{x}\left(\nu_{0} \partial_{x} V_{e q}\right)-\mu\left(V_{e q}-V_{0}\right)
\end{aligned}
$$

Notice that the nonlinear particle flux and the Reynolds stress take the following simple forms:

$$
\begin{aligned}
& \Gamma=-2 k A_{n} A_{\phi} \sin \Delta \varphi_{n \phi} \\
& \pi=-2 k A_{\phi}^{2} \partial_{x} \varphi_{\phi}
\end{aligned}
$$

The time evolution of the fluctuations is given by:

$$
\begin{aligned}
\partial_{t} A_{n}= & k A_{\phi} \partial_{x} n_{e q} \sin \Delta \varphi_{n \phi}-\left(D_{1} k^{2}+\alpha_{n} A_{n}^{2}\right) A_{n} \\
& +\partial_{x}\left(D_{1} \partial_{x} A_{n}\right)-D_{1} A_{n}\left(\partial_{x} \varphi_{n}\right)^{2} \\
\partial_{t} A_{w}= & k A_{\phi} \partial_{x}^{2} V_{e q} \sin \Delta \varphi_{w \phi}+g k \frac{A_{n}}{n_{e q}} \sin \Delta \varphi_{n w}-\left(\nu_{1} k^{2}+\alpha_{w} A_{w}^{2}\right) A_{w} \\
& +\partial_{x}\left(\nu_{1} \partial_{x} A_{w}\right)-\nu_{1} A_{w}\left(\partial_{x} \varphi_{w}\right)^{2}
\end{aligned}
$$

with the following relationship between $A_{w}$ and $A_{\phi}$ :

$$
A_{w}=\left\{\partial_{x}^{2} A_{\phi}-\left[k^{2}+\left(\partial_{x} \varphi_{\phi}\right)^{2}\right] A_{\phi}\right\} \quad\left[\cos \Delta \varphi_{w \phi}+\tan \Delta \varphi_{w \phi}\right]
$$

The phases are governed by the following equations:

$$
\begin{aligned}
\partial_{t} \varphi_{n}= & k \frac{A_{\phi}}{A_{n}} \partial_{x} n_{e q} \cos \Delta \varphi_{n \phi}-k V_{e q} \\
& +\partial_{x}\left(D_{1} \partial_{x} \varphi_{n}\right)+2 D_{1} \partial_{x}\left(\ln A_{n}\right) \partial_{x} \varphi_{n} \\
\partial_{t} \varphi_{w}= & k \frac{A_{\phi}}{A_{w}} \partial_{x}^{2} V_{e q} \cos \Delta \varphi_{w \phi}-g k \frac{A_{n}}{n_{e q} A_{w}} \cos \Delta \varphi_{n w}-k V_{e q} \\
& +\partial_{x}\left(\nu_{1} \partial_{x} \varphi_{w}\right)+2 \nu_{1} \partial_{x}\left(\ln A_{w}\right) \partial_{x} \varphi_{w}
\end{aligned}
$$

with

$$
\tan \Delta \varphi_{w \phi}=\frac{2 \partial_{x} A_{\phi} \partial_{x} \varphi_{\phi}+A_{\phi} \partial_{x}^{2} \varphi_{\phi}}{\partial_{x}^{2} A_{\phi}-\left[k^{2}+\left(\partial_{x} \varphi_{\phi}\right)^{2}\right] A_{\phi}}
$$

\section{References}

[1] J. Abiteboul, X. Garbet, V. Grandgirard, S. J. Allfrey, Ph. Ghendrih, G. Latu, Y. Sarazin, and A. Strugarek. Conservation equations and calculation of mean flows in gyrokinetics. Phys. Plasmas, 18:082503, 2011.

[2] C.J. Ajay. Study of effect of non-adiabatic passing electron dynamics on turbulent transport in fusion plasmas using gyrokinetic simulations. PhD thesis, EPFL, 2000. 
[3] S. Benkadda, P. Beyer, N. Bian, C. Figarella, O. Garcia, X. Garbet, P. Ghendrih, Y. Sarazin, and P.H. Diamond. Bursty transport in tokamak turbulence: Role of zonal flows and internal transport barriers. Nucl. Fusion, 41:995, 2001.

[4] N. Bian, S. Benkadda, O.E. Garcia, J.-V. Paulsen, and X. Garbet. The quasilinear behavior of convective turbulence with sheared flows. Phys. Plasmas, 10:1382, 2003.

[5] H. Biglari, P.H. Diamond, and P.W. Terry. Influence of sheared poloidal rotation on edge turbulence. Phys. Fluids B, 2:1, 1990.

[6] E. Caschera, G. Dif-Pradalier, Ph. Ghendrih, V. Grandgirard, Y. Asahi, N. Bouzat, P. Donnel, X. Garbet, G. Latu, C. Passeron, and Y. Sarazin. Immersed boundary conditions in global flux-driven gyrokinetic simulations. 1125:012006, 2018.

[7] J.W. Connor, T. Fukuda, X. Garbet, C. Gormezano, V. Mukhovatov, M. Wakatani, the ITB Database Group, and the ITPA Topical Group on Transport and Internal Barrier Physics. A review of internal transport barrier physics for steady-state operation of trokamaks. Nucl. Fusion, 44:R1, 2004.

[8] J.W. Connor and H.R. Wilson. A review of theories of the L-H transition. Plasma Phys. Control. Fusion, 42:R1, 2000.

[9] P.H. Diamond, S-I Itoh, K Itoh, and T.S Hahm. Zonal flows in plasma - a review. Plasma Phys. Control. Fusion, 47:R35, 2005.

[10] P.H. Diamond and Y.-B. Kim. Theory of mean poloidal flow generation by turbulence. Phys. Plasmas, 3:1626, 1991.

[11] G. Dif-Pradalier, G. Hornung, X. Garbet, Ph. Ghendrih, V. Grandgirard, G. Latu, and Y. Sarazin. The ExB staircase of magnetised plasmas. Nucl. Fusion, 57:066026, 2017.

[12] A.M. Dimits, G. Bateman, M.A. Beer, B.I. Cohen, W. Dorland, G.W. Hammett, C. Kim, J.E. Kinsey, M. Kotschenreuther, A.H. Kritz, L.L. Lao, J. Mandrekas, W.M. Nevins, S.E. Parker, A.J. Redd, D.E. Shumaker, R. Sydora, and J. Weiland. Comparisons and physics basis of tokamak transport models and turbulence simulations. Phys. Plasmas, 7:969, 2000.

[13] A.M. Dimits, W.M. Nevins, D.E. Shumaker, G.W. Hammett, T. Dannert, F. Jenko, M.J. Pueschel, W. Dorland, S.C. Cowley, J.N. Leboeuf, T.L. Rhodes, J. Candy, and C. EstradaMila. Gyrokinetic simulations of ETG and ITG turbulence. Nucl. Fusion, 47:817, 2007.

[14] N Fedorczak, Ph Ghendrih, P Hennequin, G R Tynan, P H Diamond, and P Manz. Dynamics of tilted eddies in a transversal flow at the edge of tokamak plasmas and the consequences for $\mathrm{LH}$ transition. Plasma Phys. Control. Fusion, 55:124024, 2013.

[15] A. Fujisawa, T. Ido, A. Shimizu, S. Okamura, K. Matsuoka, H. Iguchi, Y. Hamada, H. Nakano, S. Ohshima, K. Itoh, K. Hoshino, K. Shinohara, Y. Miura, Y. Nagashima, S.-I. Itoh, M. Shats, H. Xia, J.Q. Dong, L.W. Yan, K.J. Zhao, G.D. Conway, U. Stroth, A.V. Melnikov, L.G. Eliseev, S.E. Lysenko, S.V. Perfilov, C. Hidalgo, G.R. Tynan, C. Holland, P.H. Diamond, G.R. McKee, R.J. Fonck, D.K. Gupta, and P.M. Schoch. Experimental progress on zonal flow physics in toroidal plasmas. Nucl. Fusion, 47:S718, 2007.

[16] G.I. Taylor. Eddy Motion in the Atmosphere. Philos. Trans. R. Soc. London, Ser. A, 215:1, 1915.

[17] Virginie Grandgirard, Jérémie Abiteboul, Julien Bigot, Thomas Cartier-Michaud, N. Crouseilles, Guilhem Dif-Pradalier, Charles Ehrlacher, Damien Esteve, Xavier Garbet, Philippe Ghendrih, Guillaume Latu, M. Mehrenberger, Claudia Norscini, Chantal Passeron, Fabien Rozar, Yanick Sarazin, E. Sonnendrücker, A. Strugarek, and David Zarzoso. A 5D gyrokinetic full- $f$ global semi-lagrangian code for flux-driven ion turbulence simulations. Computer Physics Communications, 207:35-68, 2016.

[18] Z.B. Guo and P.H. Diamond. Zonal Flow Patterns: How Toroidal Coupling Induces Phase Jumps and Shear Layers. Phys. Rev. Lett., 117:125002, 2016.

[19] P.N. Guzdar. Shear-flow generation by drift/Rossby waves. Phys. Plasmas, 2:4174, 1995.

[20] Akira Hasegawa, Carol G. Maclennan, and Yuji Kodama. Nonlinear behavior and turbulence spectra of drift waves and Rossby waves. Phys. Fluids, 22:2122, 1979.

[21] Akira Hasegawa and Masahiro Wakatani. Self-organization of electrostatic turbulence in a 
cylindrical plasma. Phys. Rev. Lett., 59:1581, 1987.

[22] Z. Lin, T.S. Hahmn, W.W. Leen, W.M. Tang, and R.B. White. Turbulent transport reduction by zonal flows: Massively parallel simulations. Science, 281(5384):1835, 1998.

[23] C.J. McDevitt, P.H. Diamond, Ö.D. Gürcan, and T. S. Hahm. Poloidal rotation and its relation to the potential vorticity flux. Phys. Plasmas, 17:112509, 2010.

[24] F.L. Hinton and C.W. Horton. Amplitude Limitation of a Collisional Drift Wave Instability. Phys. Fluids, 14:116, 1971.

[25] Y. Sarazin and V. Grandgirard and P. Angelino and A. Casati and G. Dif-Pradalier and X. Garbet and Ph. Ghendrih and Ö. Gürcan and P. Hennequin and R. Sabot. Turbulence spectra and transport barriers in gyrokinetic simulations. Theory of Fusion Plasmas, AIP Conference Proceedings, 1069:325, 2008.

[26] F.I. Parra and P.J. Catto. Vorticity and intrinsic ambipolarity in turbulent tokamaks. Plasma Phys. Control. Fusion, 51:095008, 2009.

[27] A.G. Peeters, F. Rath, R. Buchholz, Y. Camenen, J. Candy, F.J. Casson, S.R. Grosshauser, W.A. Hornsby, D. Strintzi, and A. Weikl. Gradient-driven flux-tube simulations of ion temperature gradient turbulence close to the non-linear threshold. Phys. Plasmas, 23:082517, 2016.

[28] P.W. Terry. Suppression of turbulence and transport by sheared flow. Reviews of Modern Physics, $72: 1,2000$.

[29] M.N. Rosenbluth and V.D. Shapiro. Analytical model of the "tilting" instability. Phys. Plasmas, 1:222, 1994.

[30] Y. Sarazin, X. Garbet, Ph. Ghendrih, and S. Benkadda. Transport due to front propagation in tokamaks. Phys. Plasmas, 7:1085, 2000.

[31] Y. Sarazin and Ph. Ghendrih. Intermittent particle transport in two-dimensional edge turbulence. Phys. Plasmas, 5:4214, 1998.

[32] L. Schmitz. The role of turbulenceflow interactions in L- to H-mode transition dynamics: recent progress. Nucl. Fusion, 57:025003, 2017.

[33] A.I. Smolyakov, P.H. Diamond, and M.V. Medvedev. Role of ion diamagnetic effects in the generation of large scale flows in toroidal ion temperature gradient mode turbulence. Phys. Plasmas, 7:3987, 2000.

[34] L. Vermare, P. Hennequin, Ö.D. Gürcan, X. Garbet, C. Honoré, F. Clairet, J.C. Giacalone, P. Morel, A. Storelli, and Tore Supra Team. Poloidal asymmetries of flows in the Tore Supra tokamak. Phys. Plasmas, 25:020704, 2018.

[35] F. Wagner. A quarter-century of H-mode studies. Plasma Phys. Control. Fusion, 49:B1, 2007.

[36] Niels Winsor, John L. Johnson, and John M. Dawson. Geodesic Acoustic Waves in Hydromagnetic Systems. Physics of Fluids, 11:2448, 1968.

[37] R.C. Wolf. Internal transport barriers in tokamak plasmas. Plasma Phys. Control. Fusion, 45:R1, 2003.

[38] T.C. Xu, X.Y. Yang, Z.B. Guo, C.J. Xiao, X.G. Wang, and R.C. He. Observation of phase pattern accelerating zonal flow. Nucl. Fusion, 60:016029, 2020. 